\title{
The CKM unitarity problem: a trace of new physics at the TeV scale?
}

\author{
Benedetta Belfatto $^{1,2,3}$, Revaz Beradze ${ }^{2,4}$, Zurab Berezhiani $^{2,3, a}$ \\ ${ }^{1}$ Gran Sasso Science Institute, 67100 L’Aquila, Italy \\ 2 Dipartimento di Fisica e Chimica, Università di L'Aquila, Coppito, 67100 L'Aquila, Italy \\ 3 INFN, Laboratori Nazionali del Gran Sasso, Assergi, 67010 L'Aquila, Italy \\ ${ }^{4}$ Ivane Javakhishvili Tbilisi State University, Chavchavadze Av. 3, 0179 Tbilisi, Georgia
}

Received: 8 June 2019 / Accepted: 28 January 2020 / Published online: 19 February 2020

(C) The Author(s) 2020

\begin{abstract}
After the recent high precision determinations of $V_{u s}$ and $V_{u d}$, the first row of the CKM matrix shows more than $4 \sigma$ deviation from unitarity. Two possible scenarios beyond the Standard Model can be investigated in order to fill the gap. If a 4th non-sequential quark $b^{\prime}$ (a vector-like weak isosinglet) participates in the mixing, with $\left|V_{u b^{\prime}}\right| \sim 0.04$, then its mass should be no more than $6 \mathrm{TeV}$ or so. A different solution can come from the introduction of the gauge horizontal family symmetry $S U(3)_{\ell}$ acting between the lepton families and spontaneously broken at the scale of about $6 \mathrm{TeV}$. Since the gauge bosons of this symmetry contribute to muon decay in interference with Standard Model, the Fermi constant is slightly smaller than the muon decay constant so that unitarity is recovered. Also the neutron lifetime problem, that is about $4 \sigma$ discrepancy between the neutron lifetimes measured in beam and trap experiments, is discussed in the light of the these determinations of the CKM matrix elements.
\end{abstract}

1. The Standard Model (SM) contains three fermion families in the identical representations of the gauge symmetry $S U(3) \times S U(2) \times U(1)$ of strong and electroweak interactions. One of its fundamental predictions is the unitarity of the Cabibbo-Kobayashi-Maskawa (CKM) matrix of quark mixing in charged current

$V_{\mathrm{CKM}}=\left(\begin{array}{ccc}V_{u d} & V_{u s} & V_{u b} \\ V_{c d} & V_{c s} & V_{c b} \\ V_{d d} & V_{t s} & V_{t b}\end{array}\right)$.

Deviation from the CKM unitarity can be a signal of new physics beyond the Standard Model (BSM). The experimental precision and control of theoretical uncertainties in the determination of the elements in the first row of $V_{\mathrm{CKM}}$ are becoming sufficient for testing the condition

$\left|V_{u d}\right|^{2}+\left|V_{u s}\right|^{2}+\left|V_{u b}\right|^{2}=1$.

a e-mail: zurab.berezhiani@1ngs.infn.it (corresponding author)
Since $\left|V_{u b}\right| \simeq 0.004$ is very small, its contribution is negligible and (2) reduces essentially to the check of the Cabibbo mixing: $\left|V_{u s}\right|=\sin \theta_{C},\left|V_{u d}\right|=\cos \theta_{C}$ and $\left|V_{u s} / V_{u d}\right|=$ $\tan \theta_{C}$. In essence, this is the universality test for the $W$ boson coupling $(g / \sqrt{2}) W_{\mu}^{+} J_{L}^{\mu}+$ h.c. to the relevant part of the charged left-handed current

$J_{L}^{\mu}=V_{u d} \overline{u_{L}} \gamma^{\mu} d_{L}+V_{u s} \overline{u_{L}} \gamma^{\mu} s_{L}+\overline{v_{e}} \gamma^{\mu} e_{L}+\overline{v_{\mu}} \gamma^{\mu} \mu_{L}$

For energies smaller than $W$-boson mass this coupling gives rise to the effective current $\times$ current interactions

$$
-\frac{4 G_{F}}{\sqrt{2}} \overline{u_{L}}\left(V_{u d} \gamma_{\mu} d_{L}+V_{u s} \gamma_{\mu} s_{L}\right)\left(\overline{e_{L}} \gamma^{\mu} v_{e}+\overline{\mu_{L}} \gamma^{\mu} v_{\mu}\right)
$$

which are responsible for leptonic decays of the neutron, pions, kaons etc., as well as to the interaction

$$
-\frac{4 G_{F}}{\sqrt{2}}\left(\overline{e_{L}} \gamma_{\mu} v_{e}\right)\left(\overline{v_{\mu}} \gamma^{\mu} \mu_{L}\right)
$$

responsible for the muon decay. All these couplings contain the Fermi constant $G_{F} / \sqrt{2}=g^{2} / 8 M_{W}^{2}$.

Precision experimental data on kaon decays, in combination with the lattice QCD calculations of the decay constants and form-factors, provide accurate information about $\left|V_{u s}\right|$. On the other hand, recent calculations of short-distance radiative corrections in the neutron decay allow to determine $\left|V_{u d}\right|$ with a remarkable precision.

In this paper we analyze the present individual determinations of $V_{u d}$ and $V_{u s}$ and find significant (about $4 \sigma$ ) deviation from the CKM unitarity (2). We discuss two possible BSM scenarios which can explain this deviation. In the first one the three-family unitarity is extended to four species, by introducing the 4 th non-sequential down-type quark $b^{\prime}$ in the form of vector-like weak isosinglet with mass of few 
$\mathrm{TeV}$. The second scenario assumes the existence of horizontal gauge symmetry between the lepton families which is spontaneously broken at the scale of few TeV. The corresponding flavor changing gauge bosons induce the effective four-lepton interaction having exactly the same form as (5), with the new Fermi-like constant $G_{\mathcal{F}}$. In this case, muon lifetime would determine $G_{\mu}=G_{F}+G_{\mathcal{F}}$ rather than $G_{F}$. In this way, one can nicely restore the three family unitarity (2) without introducing new quark species. We discuss implications of these scenarios for the lepton flavor violation (LFV) and for the Standard Model precision tests. At the end, we also discuss the problem of neutron lifetime related to the discrepancy between its values measured using two different (trap and beam) methods.

2 . The most precise determination of $\left|V_{u d}\right|$ is obtained from superallowed $0^{+}-0^{+}$nuclear $\beta$-decays which are pure Fermi transitions sensitive only to the vector coupling constant $G_{V}=G_{F}\left|V_{u d}\right|[1]$ :

$\left|V_{u d}\right|^{2}=\frac{K}{2 G_{F}^{2} \mathcal{F} t\left(1+\Delta_{R}^{V}\right)}=\frac{0.97147(20)}{1+\Delta_{R}^{V}}$

where $K=2 \pi^{3} \ln 2 / m_{e}^{5}=8120.2776(9) \times 10^{-10} \mathrm{~s} / \mathrm{GeV}^{4}$ and $\mathcal{F} t$ is the nucleus independent value obtained from the individual $f t$-values of different $0^{+}-0^{+}$nuclear transitions by absorbing in the latter all nucleus-dependent corrections, while $\Delta_{R}^{V}$ accounts for short-distance (transition independent) radiative corrections. For the second step, we take $\mathcal{F} t=$ 3072.07(72) s [2] obtained by averaging the individual $\mathcal{F} t$ values for fourteen superallowed $0^{+}-0^{+}$transitions determined with the best experimental accuracy, and plug in the Fermi constant as $G_{F}=G_{\mu}=1.1663787(6) \times 10^{-5} \mathrm{GeV}^{-2}$ determined from the muon decay [3]. The major uncertainty is related to the so called inner radiative correction $\Delta_{R}^{V}$.

The element $\left|V_{u s}\right|$ can be determined from the analysis of semileptonic $K \ell 3$ decays ( $K_{L} \mu 3, K_{L} e 3, K^{ \pm} e 3$, etc.) [4]:

$f_{+}(0)\left|V_{u s}\right|=0.21654 \pm 0.00041$

where $f_{+}(0)$ is the $K \rightarrow \pi \ell v$ vector form-factor at zero momentum transfer. On the other hand, by comparing the kaon and pion inclusive radiative decay rates $K \rightarrow \mu \nu(\gamma)$ and $\pi \rightarrow \mu \nu(\gamma)$, one obtains [5]:

$\left|V_{u s} / V_{u d}\right| \times\left(f_{K^{ \pm}} / f_{\pi^{ \pm}}\right)=0.27599 \pm 0.00038$

Hence, the values $\left|V_{u s}\right|$ and $\left|V_{u s} / V_{u d}\right|$ can be independently determined using the lattice QCD results for the form-factor $f_{+}(0)$ and the decay constant ratio $f_{K} / f_{\pi}$.

3. Let us first consider the values of the CKM matrix elements $\left|V_{u s}\right|,\left|V_{u d}\right|$ and their ratio $\left|V_{u s} / V_{u d}\right|$ as quoted by Particle Data Group (PDG) review 2018 [5]:

$\left|V_{u s}\right|=0.2238(8)$

$\left|V_{u s} / V_{u d}\right|=0.2315(10)$

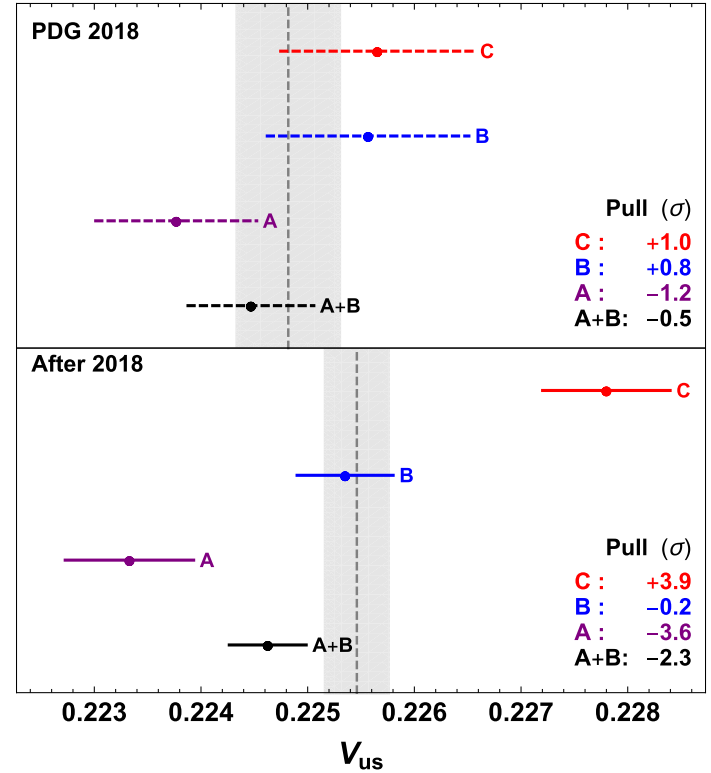

Fig. 1 Upper panel: three independent $\left|V_{u s}\right|$ determinations A, B, C obtained from the PDG 2018 data (9) by assuming the CKM unitarity. The grey shaded band corresponds to the average $A+B+C$ (with formal error not rescaled by a factor $\sqrt{\chi_{\text {dof }}^{2}}$ ). Pulls of C, B, A and A + $\mathrm{B}$ are shown. Lower panel: the same for A, B, C values obtained from the dataset (10)

$\left|V_{u d}\right|=0.97420(21)$

Here $\left|V_{u s}\right|$ and $\left|V_{u s} / V_{u d}\right|$ are obtained respectively from Eqs. (7) and (8) using the FLAG 2017 averages of 3flavor lattice QCD simulations $f_{+}(0)=0.9677(27)$ and $f_{K^{ \pm}} / f_{\pi^{ \pm}}=1.192(5)[6] .\left|V_{u d}\right|$ is obtained from Eq. (6) by taking $\Delta_{R}^{V}=0.02361$ (38) as calculated in Ref. [7].

By imposing the CKM unitarity (2), the three data (9) reduce to three independent determinations of $\left|V_{u s}\right|$. These determinations shown as A, B, C in upper panel of Fig. 1 (see also Table 1 for numerical values) are compatible within their error-bars. Throughout this paper A is the direct determination of $\left|V_{u s}\right|$ obtained from Eq. (7). B and $\mathrm{C}$ are the values of $\left|V_{u s}\right|$ obtained respectively from $\left|V_{u s} / V_{u d}\right|$ and $\left|V_{u d}\right|$ by assuming unitarity. Namely, $\mathrm{B}$ and $\mathrm{C}$ are almost equal while there is a modest tension $(1.4 \sigma)$ between $\mathrm{A}$ and $\mathrm{B}$. Their average $\overline{A+B}=0.2245$ (6) practically coincides with the PDG 2018 average of $\left|V_{u s}\right|$ [5]. By averaging all three values we get $\overline{A+B+C}=0.2248(5)$ with $\chi_{\text {dof }}^{2}=1.7$. Pulls of $\mathrm{A}, \mathrm{B}$ and $\mathrm{C}$ relative to this average (given in Fig. 1) are compatible with a standard deviation. Summarizing, the dataset (9) adopted from PDG 2018 [5] is consistent with the CKM unitarity (2).

However, recent progress in the determination of the CKM elements allows to test the unitarity with improved precision. Significant redetermination of $\left|V_{u d}\right|$ is related to new calculation of inner radiative corrections with reduced hadronic uncertainties, $\Delta_{R}^{V}=0.02467(22)$ [8]. Employing 
Table 1 The 1st column shows independent $\left|V_{u s}\right|$

determinations A, B, C from the PDG dataset (9) by assuming 3-family CKM unitarity (2), their averages and total $\chi^{2}$ value

\begin{tabular}{lllll}
\hline & CKM [PDG] & CKM [post 2018] & CKM+ $b^{\prime}$ & CKM $+\mathcal{F}$ \\
\hline $\mathrm{C}$ & $0.2257(9)$ & $0.22780(60)$ & $0.22443(61)$ & $0.22460(61)$ \\
$\mathrm{B}$ & $0.2256(10)$ & $0.22535(45)$ & $0.22518(45)$ & $0.22535(45)$ \\
$\mathrm{A}$ & $0.2238(8)$ & $0.22333(60)$ & $0.22333(60)$ & $0.22350(60)$ \\
$\overline{A+B}$ & $0.2245(6)$ & $0.22463(36)$ & $0.22452(36)$ & $0.22469(36)$ \\
$A+B+C$ & $0.2248(5)$ & $0.22546(31)$ & $0.22449(31)$ & $0.22467(31)$ \\
$\left|V_{u s}\right|$ & $\chi^{2}=3.4$ & $\chi^{2}=27.7^{\dagger}$ & $\chi^{2}=6.1$ & $\chi^{2}=6.1$ \\
$\left|V_{u d}\right|$ & $0.2248(7)$ & $0.2255(12)^{\dagger}$ & $0.2245(5)$ & $0.2247(5)$ \\
\hline
\end{tabular}

The last two rows show the conservative estimation of $\left|V_{u s}\right|$ with error-bar rescaled by $\sqrt{\chi_{\text {dof }}^{2}}$ and the corresponding value of $\left|V_{u d}\right|$. Other columns show the same but obtained from after 2018 dataset (10) by assuming respectively 3-family CKM unitarity (2), unitarity extended to 4th quark $b^{\prime}$ with $\left|V_{u b^{\prime}}\right|=0.04$, and 3-family CKM but taking $G_{\mu} / G_{F}=1+\delta_{\mu}$ with $\delta_{\mu}=7.6 \times 10^{-4}$. Mark ${ }^{\dagger}$ in 2 nd column indicates that for that large $\chi^{2}$ the error-rescaling by $\sqrt{\chi_{\text {dof }}^{2}}=3.7$ does not make much sense since the data are incompatible also the recent result $f_{+}(0)=0.9696(18)$ from new 4-flavor $\left(N_{f}=2+1+1\right)$ lattice QCD simulations [9] and the FLAG 2019 four-flavor average $f_{K^{ \pm}} / f_{\pi^{ \pm}}=1.1932$ (19) [10], one arrives to the following:

$\left|V_{u s}\right|=0.22333(60)$

$\left|V_{u s} / V_{u d}\right|=0.23130(50)$

$\left|V_{u d}\right|=0.97370(14)$

This dataset, again by imposing the CKM unitarity, reduces to independent $\left|V_{u s}\right|$ values A, B, C shown in lower panel of Fig. 1 (numerical values are given in Table 1).

Now we see that the values A, B, C are in tensions among each other. Namely, there is a $5.3 \sigma$ discrepancy between $\mathrm{A}$ and $\mathrm{C}$, and $3.2 \sigma$ between $\mathrm{B}$ and $\mathrm{C}$. The tension between the determinations $\mathrm{A}$ and $\mathrm{B}$, both from kaon physics, is $2.7 \sigma$. More conservatively, one can take their average $\overline{A+B}$. The discrepancy of the latter with $C$ is $4.5 \sigma$. Fitting these values, we get $\overline{A+B+C}=0.22546(31)$ but the fit is bad, $\chi_{\text {dof }}^{2}=$ 13.9. $\mathrm{C}, \mathrm{A}$ and $\mathrm{A}+\mathrm{B}$ have large pulls, $3.9 \sigma,-3.6 \sigma$ and $-2.3 \sigma$.

Let us remark that the chosen dataset (10) is rather indicative since there are tensions in various determinations of $\left|V_{u s}\right|$ which may disappear with more accurate lattice simulations. In particular, we have employed the latest and most precise result $f_{+}(0)=0.9696(18)$ from 4-flavor lattice QCD simulations [9] which is perfectly compatible with the FLAG 20194-flavor value $f_{+}(0)=0.9706(27)$ [10] which does not include the result of Ref. [9]. Their average yields $f_{+}(0)=$ $0.9699(15)$ which would give $\left|V_{u s}\right|=0.22326(55)$. However, this result from $K \ell 3$ decays is discordant with the independent determination $\left|V_{u s}\right|=0.22567(42)$ from $K \mu 2$ decays recently reported in Ref. [11]. Therefore, for determination A one can take a conservative average between these two results, $\left|V_{u s}\right|=0.22478(69)$, where the errors are quadratically combined because of their poor compatibility.

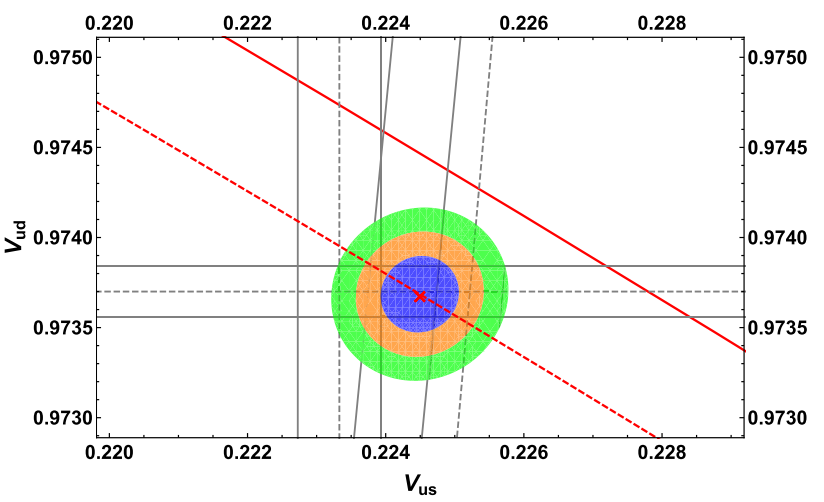

Fig. 2 The horizontal, vertical and slightly bended bands correspond to $\left|V_{u d}\right|,\left|V_{u s}\right|$ and $\left|V_{u s} / V_{u d}\right|$ from (10). The best fit point (red cross) and 1,2 and $3 \sigma$ contours are shown. The red solid line corresponds to the three family unitarity condition (2), and the dashed red line corresponds to the "extended" unitarity (11) with $\left|V_{u b^{\prime}}\right|=0.04$

Let us remark also that the latter determination $\mathrm{A}$ is well compatible with the determination $\mathrm{B}\left|V_{u s}\right|=0.22535$ (45) deduced from $\left|V_{u s} / V_{u d}\right|$ given in (10), so that for the average $\overline{A+B}$ we get $\left|V_{u s}\right|=0.22518(37)$.

Regarding the determination of $\left|V_{u d}\right|$, we adopted the result $\Delta_{R}^{V}=0.02467(22)$ of Ref. [8] which is mildly different from the value $\Delta_{R}^{V}=0.02426(32)$ deduced in Ref. [12]. For being more conservative, we can average these two results as $\Delta_{R}^{V}=0.02454(32)$, without reducing the largest uncertainty. In doing so, from Eq. (6) we obtain $\left|V_{u d}\right|=0.97376(10)_{\mathcal{F}_{t}}(10)_{\Delta_{R}^{V}}=0.97376(14)$ which in turn gives determination $\mathrm{C}$ as $\left|V_{u s}\right|=0.22756(72)$. Therefore, between the determination $\mathrm{C}$ and $A+B$ remains $3 \sigma$ tension even with more conservative treatment.

Let us analyze our dataset (10) also in a different way. Without imposing the unitarity condition (2), we perform a two parameter fit of the three independent values (10). In Fig. 2 we show the gaussian hill of the probability distribution 
with the confidence level (C.L.) contours around the best fit point $\left(\left|V_{u s}\right|=0.22449,\left|V_{u d}\right|=0.97369\right)$, with $\chi_{\min }^{2}=6.1$. (This $\chi^{2}$-value seems large for a two parameter fit, but it is dominated by the tension between $\left|V_{u s}\right|$ determinations $\mathrm{A}$ and $\mathrm{B}$ from the kaon data which may be not real as we discussed above.) The red solid line corresponding to the three family unitarity condition $\left|V_{u d}\right|^{2}+\left|V_{u s}\right|^{2}=1-\left|V_{u b}\right|^{2}=1-$ $O\left(10^{-5}\right)$ is about $4.3 \sigma$ away from this hill top $\left(\Delta \chi^{2}=21.6\right)$ which means that the new (after 2018) dataset (10) disfavors the CKM unitarity at $99.998 \%$ C.L.

4. "If the Hill does not come to the CKM, the CKM will go to the Hill." The unitarity line can be moved down towards the probability distribution hill in Fig. 2 if the unitarity condition is extended to more families.

In fact, by introducing just one additional (fourth) family which is also involved in quark mixing, the first row unitarity condition (2) will be modified to

$\left|V_{u d}\right|^{2}+\left|V_{u s}\right|^{2}+\left|V_{u b}\right|^{2}=1-\left|V_{u b^{\prime}}\right|^{2}$.

In particular, the red dashed line in Fig. 2 passing through the best fit point on the top of the probability hill corresponds to $\left|V_{u b^{\prime}}\right|=0.04$ (at 95\% C.L. this additional mixing is limited as $\left|V_{u b^{\prime}}\right|=0.04 \pm 0.01$ ). Plugging this value in Eq. (11), the dataset (10) gives the modified determinations of $\left|V_{u s}\right|$ for the three cases named above as A, B and C (for numerical values see in 3rd column of Table 1). Clearly, the case A in this list remains the same as in 2 nd column but $B$ and especially $C$ are shifted down. Figure 3 shows that consistency between these values is significantly improved compared to lower panel of Fig. 1. The fit for $\overline{A+B+C}$ is acceptable, $\chi_{\text {dof }}^{2}=3$. Pulls of $\mathrm{C}$ and $\mathrm{A}+\mathrm{B}$ are practically vanishing. There remains a tension between $\mathrm{A}$ and $\mathrm{B}$ but it is softened to $2.4 \sigma$ from $2.7 \sigma$ of Fig. 1.

In the SM the three families $(i=1,2,3$ is the family index) of left-handed (LH) quarks $Q_{L i}=\left(u_{i}, d_{i}\right)_{L}$ and leptons $\ell_{L i}=\left(v_{i}, e_{i}\right)_{L}$ transform as weak isodoublets of $S U(2) \times U(1)$ and the right-handed (RH) quarks $u_{R i}, d_{R i}$ and leptons $e_{R i}$ are isosinglets. Their masses emerge from the Yukawa couplings with the Higgs doublet $\phi$ :

$Y_{u}^{i j} \tilde{\phi} \overline{Q_{L i}} u_{R j}+Y_{d}^{i j} \phi \overline{Q_{L i}} d_{R j}+Y_{e}^{i j} \phi \overline{\ell_{L i}} e_{R j}+$ h.c.

$Y_{u, d, e}$ being the Yukawa constant matrices and $\tilde{\phi}=i \tau_{2} \phi^{*}$.

The existence of a fourth sequential family is excluded by the SM precision tests in combination with the lower limits on their masses from the LHC, as well as from the LHC data on the Higgs production via gluon fusion and and its decay in $2 \gamma$ [13]. However, one can introduce additional vector-like fermions.

Let us briefly sketch a simple picture of this type introducing just an additional vector-like couple of isosinglet down-type quarks $d_{4 L}, d_{4 R}$. Since 4 species of RH quarks $d_{1 R}, d_{2 R}, d_{3 R}, d_{4 R}$ have identical quantum numbers, $d_{4 R}$ can

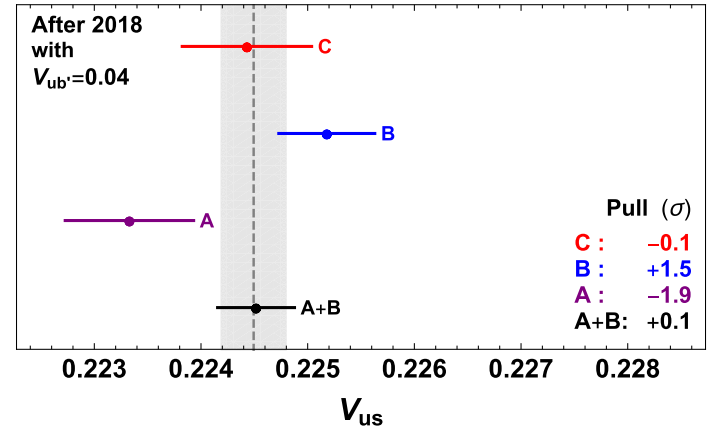

Fig. 3 Determinations of $\left|V_{u s}\right|$ obtained from the dataset (10) using Eq. (11) with $\left|V_{u b^{\prime}}\right|=0.04$

be identified as their combination which makes a mass term $M$ with isosinglet LH state $d_{4 L}$. Then, besides the standard Yukawa terms (12) the Lagrangian should contain the additional terms

$Y_{d}^{i 4} \phi \overline{Q_{L i}} d_{4 R}+M \overline{d_{4 L}} d_{4 R}+$ h.c.

Fermion masses of three normal (chiral) families emerge from the vacuum expectation value (VEV) of the Higgs, $\left\langle\phi^{0}\right\rangle=v_{\mathrm{w}}=174 \mathrm{GeV}$ (for a convenience, we use this normalization of the Higgs VEV instead of "standard" normalization $\langle\phi\rangle=v / \sqrt{2}$, i.e. $v=\sqrt{2} v_{\mathrm{w}}$ ). Without loss of generality, the $3 \times 3$ Yukawa matrix $Y_{u}$ of up quarks can be chosen diagonal, $Y_{u}=Y_{u}^{\text {diag }}=\operatorname{diag}\left(y_{u}, y_{c}, y_{t}\right)$, in which bases the states $u_{1,2,3}$ coincide with the mass eigenstates $u, c, t$ so that $m_{t}=y_{t} v_{\mathrm{w}}$, etc. In this basis the Yukawa matrix $Y_{d}^{i j}$ is non-diagonal, and in addition the terms in (13) induce the mixing of three known down quarks with the 4th species. Thus, $4 \times 4$ mass matrix of all down-type quarks has a form:

$\mathcal{M}=\left(\begin{array}{cc}Y_{d}^{i j} v_{\mathrm{w}} & Y_{d}^{i 4} v_{\mathrm{w}} \\ 0 & M\end{array}\right)$.

It can be diagonalized by bi-unitary transformation $\mathcal{M} \rightarrow$ $\mathcal{M}_{\text {diag }}=V_{L}^{\dagger} \mathcal{M} V_{R}$ where $4 \times 4$ unitary matrices $V_{L, R}$ connect $d_{1,2,3,4}$ with the mass eigenstates $d, s, b, b^{\prime}$ :

$$
\left(\begin{array}{l}
d_{1} \\
d_{2} \\
d_{3} \\
d_{4}
\end{array}\right)_{L, R}=\left(\begin{array}{llll}
V_{1 d} & V_{1 s} & V_{1 b} & V_{1 b^{\prime}} \\
V_{2 d} & V_{2 s} & V_{2 b} & V_{2 b^{\prime}} \\
V_{3 d} & V_{3 s} & V_{3 b} & V_{3 b^{\prime}} \\
V_{4 d} & V_{4 s} & V_{4 b} & V_{4 b^{\prime}}
\end{array}\right)_{L, R}\left(\begin{array}{c}
d \\
s \\
b \\
b^{\prime}
\end{array}\right)_{L, R}
$$

In the context of the SM, the mixing $V_{R}$ of the RH quarks is not of interest. As for the left-handed charged current we obtain the modified $3 \times 4 \mathrm{CKM}$ mixing matrix describing $W$ boson interactions between three up quarks $u, c, t$ and four down quarks $d, s, b, b^{\prime}$ :

$$
\tilde{V}_{\mathrm{CKM}}=\left(\begin{array}{cccc}
V_{u d} & V_{u s} & V_{u b} & V_{u b^{\prime}} \\
V_{c d} & V_{c s} & V_{c b} & V_{c b^{\prime}} \\
V_{t d} & V_{t s} & V_{t b} & V_{t b^{\prime}}
\end{array}\right)
$$


which in fact consists of three upper rows in $4 \times 4$ unitary matrix $V_{L}$ (15), i.e. $V_{u d}=V_{L 1 d}, V_{u s}=V_{L 1 s}$ etc. Then the condition (11) regards the first row of this matrix and it stems from the unitarity of $V_{L}$. As for the first column, we have $\left|V_{u d}\right|^{2}+\left|V_{c d}\right|^{2}+\left|V_{t d}\right|^{2}=1-\left|V_{4 d}\right|^{2}$ where $V_{4 d}$ is the 4th row element in matrix $V_{L}$ which is "deleted" for transforming the latter into $\tilde{V}_{\mathrm{CKM}}$.

Let us discuss now in which conditions one could obtain large enough mixing with the 4th species, $\left|V_{u b^{\prime}}\right|=0.04$ or so. From the structure of mass matrix (14), for $M \gg v_{\mathrm{w}}$ we have $\left|V_{u b^{\prime}}\right| \approx Y_{d}^{14} v_{\mathrm{w}} / M$. Then the LHC limit on extra $b^{\prime}$ mass $M>880 \mathrm{GeV}$ [5] implies that $\left|V_{u b^{\prime}}\right| \simeq 0.04$ can be obtained if the coupling constant $Y_{d}^{14}$ in (13) is larger than 0.2 or so. In other words, it should be much larger than the Yukawa constant $y_{b}$ of the bottom quark. In turn, by taking $\left|V_{u b^{\prime}}\right|>0.03$ and assuming (for the perturbativity) $Y_{d}^{14}<y_{t} \simeq 1$, we get an upper limit on the extra quark mass, $M<6 \mathrm{TeV}$ or so. Thus, the mass of the extra state $b^{\prime}$ should be in the range of few $\mathrm{GeV}$.

The CKM unitarity (2) can be also corrected by introducing a 4th up quark $t^{\prime}$ instead of the 4th down quark $b^{\prime}$, or more generically by introducing both $b^{\prime}$ and $t^{\prime}$ forming in some sense a complete vector-like 4th family. In the latter case, the mixing matrix (16) would become a $4 \times 4$ matrix, however it will not be unitary as far as $b^{\prime}$ and $t^{\prime}$ states are weak isosinglets. Interestingly, it can be shown that introduction of a fourth vector-like isodoublet family $Q_{L, R}^{\prime}=\left(t^{\prime}, b^{\prime}\right)_{L, R}$ can also have large enough effect for smoothing the discrepancies between the mixing angle determinations [14].

One has to remark, however, that the mixing of ordinary quarks with 4th species induces the quark flavor changing couplings of $Z$-boson at the tree-level. In the case of extra isosinglet down quark $b^{\prime}$ this question was discussed in Ref. [15]. In fact, $Z$ boson couples the neutral current of fermions $J_{\mathrm{nc}}^{\mu}=\sum_{f} \overline{f_{L, R}}\left[I_{3}(f)-\sin ^{2} \theta_{W} Q(f)\right] \gamma^{\mu} f$ where $I_{3}$ and $Q$ are respectively weak isospin and electric charge of a fermion $f$, and $\theta_{W}$ is the Weinberg angle. In the case of down quarks $d_{1,2,3,4}$ the second part proportional to $Q=-1 / 3$ is uniform for all four states of the LH and RH chirality, and thus it reduces to a flavor-diagonal (and vector) current also in the mass basis $d, s, b, b^{\prime}$, as a result of the unitarity of the matrices $V_{L}$ and $V_{R}$. For the RH states also the isospin dependent part is uniform since $d_{i R}, i=1, \ldots 4$, all have the same isosipns $I_{3}=0$ and thus their mixing $V_{R}$ cannot induce any flavor-changing couplings of $Z$ in the mass basis $\left(d, s, b, b^{\prime}\right)_{R}$.

However, the four LH states have different isospins, namely $d_{1 L}, d_{2 L}, d_{3 L}$ have $I_{3}=-1 / 2$ while $d_{4 L}$ has $I_{3}=0$. Therefore, in the initial basis $\left(d_{1}, d_{2}, d_{3}, d_{4}\right)_{L}$ the isospin part in the neutral current is represented by the matrix $\tilde{I}=\frac{1}{2} \operatorname{diag}(1,1,1,0)$. Therefore, after rotating to the mass basis by a matrix $V_{L}$, the isospin part induces non-standard
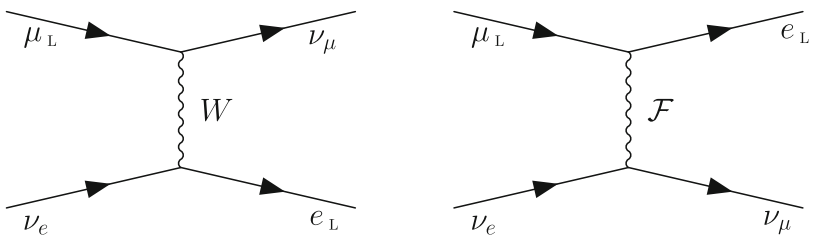

Fig. 4 The SM contribution to the muon decay mediated by $W$-boson (left), and the BSM contribution mediated by the flavor-changing $\mathcal{F}-$ boson (right)

couplings of $Z$ boson to the $\mathrm{LH}$ states described by the matrix $V_{\mathrm{NS}}=V_{L}^{\dagger} \operatorname{diag}(0,0,0,1) V_{L}$, or explicitly

$V_{\text {NS }}=\left(\begin{array}{cccc}\left|V_{4 d}\right|^{2} & V_{4 d}^{*} V_{4 s} & V_{4 d}^{*} V_{4 b} & V_{4 d}^{*} V_{4 b^{\prime}} \\ V_{4 s}^{*} V_{4 d} & \left|V_{4 s}\right|^{2} & V_{4 s}^{*} V_{4 b} & V_{4 s}^{*} V_{4 b^{\prime}} \\ V_{4 b}^{*} V_{4 d} & V_{4 b}^{*} V_{4 s} & \left|V_{4 b}\right|^{2} & V_{4 b}^{*} V_{4 b^{\prime}} \\ V_{4 d} & V_{4 s} & V_{4 b} & V_{4 b^{\prime}}\end{array}\right)_{L}$

These couplings can induce strong flavor-changing and CP-violating effects in $K^{0}-\bar{K}^{0}$ system, as well as too large decay rates for $K_{L} \rightarrow \mu^{+} \mu^{-}$etc. [15]. In fact, they can be suppressed if $V_{c b^{\prime}}$ and $V_{t b^{\prime}}$ are much less than $V_{u b^{\prime}}$, or at least have rather small complex parts. (Accidentally, $\left|V_{u b^{\prime}}\right| \simeq 0.04$ is comparable to $\left|V_{c b}\right|$ and ten times larger than $\left|V_{u b}\right|$.) The picture with the 4th state $b^{\prime}$ having a larger mixing with the first family than with (heavier) 2nd and 3rd families looks somewhat $a d$ hoc, but it is not excluded by the present experimental limits. The implications of a $\mathrm{TeV}$ scale extra vectorlike quarks $b^{\prime}$ or $t^{\prime}$ with significant mixing with the three normal families deserve careful analysis which will be given in details elsewhere [14].

5. "But what if the Hill comes to the CKM?" Here we discuss just the opposite possibility: instead of moving the unitarity line to the probability distribution Hill in Fig. 2, we move the Hill towards the unitarity line.

Namely, we consider that the Fermi constant $G_{F}$ in the effective interaction (4) which is responsible for leptonic decays of hadrons can be different from the effective constant $G_{\mu}$ determined from the muon lifetime. We assume that besides the SM interaction (5) mediated by charged $W$ boson, there is also a new operator

$-\frac{4 G_{\mathcal{F}}}{\sqrt{2}}\left(\overline{e_{L}} \gamma_{\mu} \mu_{L}\right)\left(\overline{v_{\mu}} \gamma^{\mu} v_{e}\right)$

mediated by a hypothetical lepton flavor changing neutral gauge boson $\mathcal{F}$. The respective diagrams, shown in Fig. 4, have positive interference for the muon decay. Namely, by Fierz transformation this new operator can be brought to the form (5), so that the sum of these two diagrams effectively gives the operator

$-\frac{4 G_{\mu}}{\sqrt{2}}\left(\overline{e_{L}} \gamma_{\mu} v_{e}\right)\left(\overline{v_{\mu}} \gamma^{\mu} \mu_{L}\right)$ 


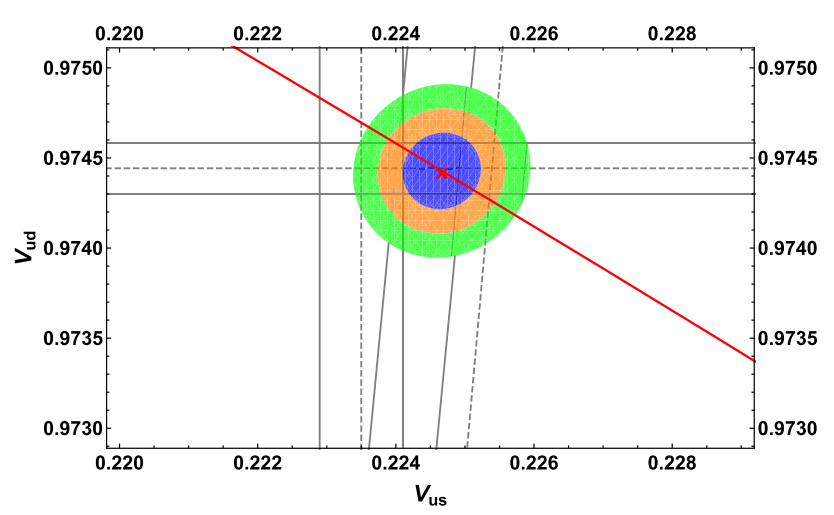

Fig. 5 The same as on Fig. 2 but with the bands of $\left|V_{u d}\right|,\left|V_{u s}\right|$ and $\left|V_{u d} / V_{u s}\right|$ taken as in (21) with $1+\delta_{\mu}=1.00076$. The red line corresponds to 3-family unitarity (2) as in Fig. 2

the same as (5) but with the coupling constant

$G_{\mu}=G_{F}+G_{\mathcal{F}}=G_{F}\left(1+\delta_{\mu}\right), \quad \frac{G_{\mathcal{F}}}{G_{F}} \equiv \delta_{\mu}>0$.

Constant $G_{\mu}=1.1663787(6) \times 10^{-5} \mathrm{GeV}^{-2}$ is determined with great precision from the muon decay [3]. Now Eqs. (6) and (7), instead of $\left|V_{u d}\right|$ and $\left|V_{u s}\right|$, are determining respectively the values $\left|V_{u d}\right| \times G_{F} / G_{\mu}$ and $\left|V_{u s}\right| \times G_{F} / G_{\mu}$. Instead the value of $\left|V_{u s} / V_{u d}\right|$ determined from (8) remains unchanged since the Fermi constant cancels out. Thus, under our hypothesis, the dataset (10) should be modified to the following:

$\left|V_{u s}\right|=0.22333(60) \times\left(1+\delta_{\mu}\right)$

$\left|V_{u s} / V_{u d}\right|=0.23130(50)$

$\left|V_{u d}\right|=0.97370(14) \times\left(1+\delta_{\mu}\right)$

Now, involving the extra parameter $\delta_{\mu}$ but assuming the 3family unitarity (2), the fit of the above dataset has acceptable quality, $\chi^{2}=6.1$, and the best fit point corresponds to $\delta_{\mu}=$ 0.00076. This situation is shown in Fig. 5 in which the values of $\left|V_{u d}\right|$ and $\left|V_{u s}\right|$ are determined by taking $\delta_{\mu}=0.00076$. By this choice of the extra parameter the fit becomes perfectly compatible with the unitarity (2). The probability distribution Hill is moved up so that its top now lies on the unitarity line.

By imposing the unitarity condition $\left|V_{u d}\right|^{2}+\left|V_{u s}\right|^{2}=$ $1-\left|V_{u b}\right|^{2}$, the list (21) can be transformed in $\delta_{\mu}$ dependent determinations $\mathrm{A}, \mathrm{B}, \mathrm{C}$ of $\left|V_{u s}\right|$. Figure 6 shows these determinations for $\delta_{\mu}=0.00076$. Taking into account that $G_{F} / \sqrt{2}=g^{2} / 8 M_{W}^{2}=1 / 4 v_{\mathrm{w}}^{2}$, where $v_{\mathrm{w}}=174 \mathrm{GeV}$ is the weak scale, and parametrizing similarly $G_{\mathcal{F}} / \sqrt{2}=1 / 4 v_{\mathcal{F}}^{2}$, we see that $\delta_{\mu}=G_{\mathcal{F}} / G_{F}=0.00076$ corresponds to $v_{\mathcal{F}} / v_{\mathrm{W}}=36.3$, or to the flavor symmetry breaking scale $v_{\mathcal{F}}=6.3 \mathrm{TeV}$. More widely, the $1 \sigma$ interval of the parameter $\delta_{\mu}$ consistent with unitarity at the $68 \%$ C.L. is $\delta_{\mu}=$ $(7.6 \pm 1.6) \times 10^{-4}$ which corresponds to the new scale in the interval $v_{\mathcal{F}}=[5.7 \div 7.1] \mathrm{TeV}$.

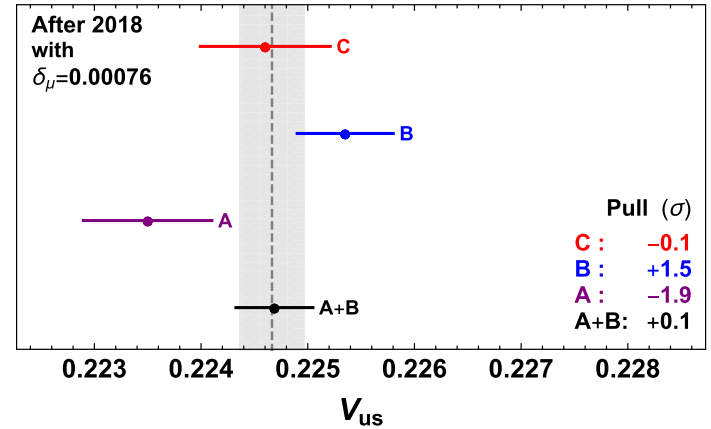

Fig. 6 Determinations of $\left|V_{u s}\right|$ obtained from (21)

6. The non-abelian gauge horizontal flavor symmetry $G_{H}$ between the fermion families can be the key for understanding the quark and lepton mass and mixing pattern [16-20]. Namely, the form of the Yukawa matrices $Y_{u, d, e}$ in (12) can be determined by the $G_{H}$ symmetry breaking pattern, i.e. by the VEV structure of the horizontal scalar fields (flavons) responsible for this breaking. Then the fermion mass hierarchy is related to the hierarchy between these VEVs. In Refs. $[16,17]$ this conjecture was coined as hypothesis of horizontal hierarchies (HHH). In this picture the fermion masses emerge from the higher order operators involving, besides the Higgs doublet $\phi$, also flavon scalars which transfer their VEV structure to the Yukawa matrices $Y_{u, d, e}$. These so called "projective" operators in the UV-complete renormalizable theory can be obtained via integrating out some extra heavy fields, scalars [18-20] or vector-like fermions [16,17]. In particular, this concept implies that the fermion masses cannot emerge if $G_{H}$ symmetry is unbroken. Thus, $G_{H}$ cannot be a vector-like symmetry but it should have a chiral character transforming the LH and RH particle species in different representations. In particular, in Refs. [16-26] the horizontal symmetry $G_{H}$ was considered as $S U(3)_{H}$ with the $\mathrm{LH}$ fermions of the three families transforming as triplets and the $\mathrm{RH}$ ones as anti-triplets, as it is motivated by the grand unification.

However, in the Standard Model framework one has more possibilities. Namely, in the limit of vanishing Yukawa couplings $Y_{u, d, e} \rightarrow 0$ in (12), the SM Lagrangian acquires a maximal global chiral symmetry $U(3)_{Q} \times U(3)_{u} \times U(3)_{d} \times$ $U(3)_{\ell} \times U(3)_{e}$ under which fermion species $Q, u$ etc. transform as triplets of independent $U(3)$ groups. It is tempting to consider that the non-abelian $S U$ (3) factors of this maximal flavor symmetry are related to gauge symmetries. ${ }^{1}$

\footnotetext{
${ }^{1}$ Gauging of chiral $U(1)$ factors is problematic because of anomalies. In fact, one combination of $U(1)$ factors can be rendered practicable via the Green-Schwarz mechanism and there are fermion mass models in which such anomalous gauge symmetry $U(1)_{A}$ is used as a flavor symmetry [27-30].
} 
Let us concentrate on the lepton sector and discuss the gauge symmetry $S U(3)_{\ell} \times S U(3)_{e}[31]$ under which the $\mathrm{LH}$ and RH lepton fields transform as

$\ell_{L \alpha}=\left(\begin{array}{c}v_{\alpha} \\ e_{\alpha}\end{array}\right)_{L} \sim\left(\mathbf{3}_{\ell}, 1\right), \quad e_{R \gamma} \sim\left(1, \mathbf{3}_{e}\right)$

where $\alpha=1,2,3$ and $\gamma=1,2,3$ are the indices of $S U(3)_{\ell}$ and $S U(3)_{e}$ respectively. This set of fermions is not anomaly free. The ways of the anomaly cancellation were discussed in Ref. [31] and in this letter we shall not concentrate on this issue. $^{2}$

For breaking $S U(3)_{\ell} \times S U(3)_{e}$ we introduce flavon fields, three triplets $\eta_{i \alpha}$ of $S U(3)_{\ell}$ and three triplets $\xi_{i \gamma}$ of $S U(3)_{e}$, $i=1,2,3$. Then the charged lepton masses emerge from the gauge invariant dimension- 6 operator

$\frac{y_{i j}}{\mathcal{M}^{2}} \eta_{i \alpha} \bar{\xi}_{j}^{\gamma} \phi \overline{\ell_{L \alpha}} e_{R \gamma}+$ h.c.

where $y_{i j}$ are order one constants, $\phi$ is the Higgs doublet and $\mathcal{M}$ is a cutoff scale. In an UV-complete theory such operators can be induced via seesaw-like mechanism by integrating out some heavy scalar or fermion states [16-20]. However, concrete model building is not the scope of this paper, and for our demonstration effective operator analysis is sufficient. As for the neutrinos, their Majorana masses are induced by the higher order operator

$\frac{h_{i j}}{\mathcal{M}_{v}^{3}} \bar{\eta}_{i}^{\alpha} \bar{\eta}_{j}^{\beta} \phi \phi \ell_{L \alpha}^{T} C \ell_{\beta}+$ h.c.

where $h_{i j}=h_{j i}$. The cutoff scale $\mathcal{M}_{\nu}$ of this operator is not necessarily the same as the scale $\mathcal{M}$ of operator (23).

In order to generate non-zero masses of all three leptons $e, \mu, \tau$, all three $S U(3)_{\ell}$ flavons $\eta_{i}$ as well as $S U(3)_{e} \xi_{i}$ should have non-zero VEVs with disoriented directions. This means that the VEVs $\left\langle\eta_{i \alpha}\right\rangle$ should form a rank-3 matrix. Without losing generality, the flavon basis can be chosen so that the matrix $\left\langle\eta_{i \alpha}\right\rangle$ is diagonal, $\left\langle\eta_{i \alpha}\right\rangle=w_{i} \delta_{i \alpha}$, i.e. the flavon VEVs are orthogonal:

$\left\langle\eta_{1}\right\rangle=\left(\begin{array}{c}w_{1} \\ 0 \\ 0\end{array}\right), \quad\left\langle\eta_{2}\right\rangle=\left(\begin{array}{c}0 \\ w_{2} \\ 0\end{array}\right), \quad\left\langle\eta_{3}\right\rangle=\left(\begin{array}{c}0 \\ 0 \\ w_{3}\end{array}\right)$

Analogously, for $\xi$-flavons we take $\left\langle\xi_{i \gamma}\right\rangle=v_{i} \delta_{i \gamma}$. After plugging these VEVs into (23) we obtain the leptonic Yukawa matrices in the SM Lagrangian (12) as

$Y_{e}^{i j}=y_{i j} \frac{w_{i} v_{j}}{\mathcal{M}^{2}}$

\footnotetext{
${ }^{2}$ One could consider also the case of vector-like horizontal symmetry $S U(3)_{V}$ under which both $\ell_{L}$ and $e_{R}$ (and RH neutrinos $N_{R}$ ) all transform as a triplet, or its $S U(2)$ subgroup. Such a symmetry is anomaly-free and it also has a custodial property for suppression of flavor-changing [32] discussed in this section. However, it allows a degenerate spectrum between the fermion families in the exact symmetry limit, and thus does not meet the paradigm of $\mathrm{HHH}$.
}

Since the couplings (23) should give the lepton mass hierarchy, we consider that the latter emerges due to the VEV hierarchy $v_{3} \gg v_{2} \gg v_{1}$ in $S U(3)_{e}$ symmetry breaking, i.e. $v_{3}: v_{2}: v_{1} \sim m_{\tau}: m_{\mu}: m_{e}$ as it is described in Ref. [31]. On the other hand, operator (24) should give the observed neutrino mass pattern, $m_{v}^{i j}=h_{i j} w_{i} w_{j} v_{\mathrm{w}}^{2} / \mathcal{M}_{v}^{3}$, and in particular the large neutrino mixing. This implies that $S U(3)_{\ell}$ breaking flavons $\eta$ should have comparable VEVs, $w_{3} \sim w_{2} \sim w_{1}$.

Gauge bosons $\mathcal{F}_{a}^{\mu}$ of $S U(3)_{\ell}$ associated to the Gell-Mann matrices $\lambda_{a}, a=1,2, \ldots 8$, interact as $g \mathcal{F}_{a}^{\mu} J_{a \mu}$ with the respective currents $J_{a \mu}=J_{a \mu}^{(e)}+J_{a \mu}^{(\nu)}=\frac{1}{2} \overline{\boldsymbol{e}_{L}} \gamma_{\mu} \lambda_{a} \boldsymbol{e}_{L}+$ $\frac{1}{2} \overline{\boldsymbol{v}_{L}} \gamma_{\mu} \lambda_{a} \boldsymbol{v}_{L}$, where $g$ is the gauge coupling constant, $\boldsymbol{e}_{L}=$ $\left(e_{1}, e_{2}, e_{3}\right)_{L}^{T}$ and $\boldsymbol{v}_{L}=\left(v_{1}, v_{2}, v_{3}\right)_{L}^{T}$ respectively denote the family triplets of the LH charged leptons and neutrinos.

At low energies these couplings induce four-fermion (current $\times$ current) interactions:

$\mathcal{L}_{\text {eff }}=-\frac{g^{2}}{2} J_{a}^{\mu}\left(M^{2}\right)_{a b}^{-1} J_{b \mu}$

where $M_{a b}^{2}$ is the squared mass matrix of gauge bosons $\mathcal{F}_{a}^{\mu}$ which in the flavon VEV basis (25) is essentially diagonal apart of a non-diagonal $2 \times 2$ block related to $\mathcal{F}_{3}^{\mu}-\mathcal{F}_{8}^{\mu}$ mixing. Namely, the masses of $\mathcal{F}_{1,2}^{\mu}, \mathcal{F}_{4,5}^{\mu}$ and $\mathcal{F}_{6,7}^{\mu}$ are

$$
\begin{aligned}
& M_{1,2}^{2}=\frac{g^{2}}{2}\left(w_{2}^{2}+w_{1}^{2}\right)=\frac{g^{2}}{2} v_{\mathcal{F}}^{2}, \\
& M_{4,5}^{2}=\frac{g^{2}}{2}\left(w_{3}^{2}+w_{1}^{2}\right), \quad M_{6,7}^{2}=\frac{g^{2}}{2}\left(w_{3}^{2}+w_{2}^{2}\right) .
\end{aligned}
$$

As for $\mathcal{F}_{3}^{\mu}$ and $\mathcal{F}_{8}^{\mu}$ they have a mass mixing and their mass matrix reads

$M_{38}^{2}=\frac{g^{2}}{2}\left(\begin{array}{cc}w_{2}^{2}+w_{1}^{2} & \frac{1}{\sqrt{3}}\left(w_{1}^{2}-w_{2}^{2}\right) \\ \frac{1}{\sqrt{3}}\left(w_{1}^{2}-w_{2}^{2}\right) & \frac{1}{3}\left(4 w_{3}^{2}+w_{1}^{2}+w_{2}^{2}\right)\end{array}\right)$.

Notice that if $w_{1}=w_{2}=v_{\mathcal{F}} / \sqrt{2}$, this matrix becomes diagonal. In the following, for the simplicity of our demonstration, we analyze this case. ${ }^{3}$ Then for the gauge boson masses we have $M_{a}^{2}=\left(g^{2} / 2\right)\left(x_{a} v_{\mathcal{F}}\right)^{2}$, where

$x_{1,2,3}^{2}=1, \quad x_{4,5,6,7}^{2}=\frac{r+1}{2}, \quad x_{8}^{2}=\frac{2 r+1}{3}$

and $r=2 w_{3}^{2} / v_{\mathcal{F}}^{2}$. Then operators (27) can be rewritten as $\mathcal{L}_{\text {eff }}=\mathcal{L}_{\text {eff }}^{e v}+\mathcal{L}_{\text {eff }}^{e e}+\mathcal{L}_{\text {eff }}^{v v}$ where

$$
\begin{aligned}
& \mathcal{L}_{\text {eff }}^{e v}=-\frac{2 G_{\mathcal{F}}}{\sqrt{2}} \sum_{a=1}^{8}\left(\overline{\boldsymbol{e}_{L}} \gamma^{\mu} \frac{\lambda_{a}}{x_{a}} \boldsymbol{e}_{L}\right)\left(\overline{\boldsymbol{v}_{L}} \gamma_{\mu} \frac{\lambda_{a}}{x_{a}} \boldsymbol{v}_{L}\right) \\
& \mathcal{L}_{\mathrm{eff}}^{e e}=-\frac{G_{\mathcal{F}}}{\sqrt{2}} \sum_{a=1}^{8}\left(\overline{\boldsymbol{e}_{L}} \gamma_{\mu} \frac{\lambda_{a}}{x_{a}} \boldsymbol{e}_{L}\right)^{2}
\end{aligned}
$$

\footnotetext{
3 Similar analysis can be done also for a general case $w_{1} \neq w_{2}$, along the lines of Ref. [31] where such analysis was done for the RH gauge sector $S U(3)_{e}$.
} 


$$
\mathcal{L}_{\text {eff }}^{v v}=-\frac{G_{\mathcal{F}}}{\sqrt{2}} \sum_{a=1}^{8}\left(\overline{\boldsymbol{v}_{L}} \gamma_{\mu} \frac{\lambda_{a}}{x_{a}} \boldsymbol{v}_{L}\right)^{2}
$$

where $4 G_{\mathcal{F}} / \sqrt{2}=1 / v_{\mathcal{F}}^{2}$. Obviously, the factor $g^{2} / 2$ in operators cancels out and the strength of these operators is determined solely by the VEVs (25).

The first term $\mathcal{L}_{\text {eff }}^{e v}$ contains operator (18) which contributes to the muon decay $\mu \rightarrow e v_{\mu} \bar{\nu}_{e}$ as $G_{\mu}=G_{F}+G_{\mathcal{F}}$. It is induced by exchange of gauge bosons $\mathcal{F}_{1}^{\mu}$ and $\mathcal{F}_{2}^{\mu}$, or more precisely by the combination $\left(\mathcal{F}_{1}^{\mu} \pm i \mathcal{F}_{2}^{\mu}\right) / \sqrt{2}$, as in second diagram of Fig. 4. As it was pointed out in the previous section, for restoring the CKM unitarity one needs $\delta_{\mu}=G_{\mathcal{F}} / G_{F}=\left(v_{\mathrm{w}} / v_{\mathcal{F}}\right)^{2}$ to be around $7 \times 10^{-4}$ which corresponds to the flavor scale $v_{\mathcal{F}}=6 \div 7 \mathrm{TeV}$.

The similar operators in $\mathcal{L}_{\text {eff }}^{e v}$ mediated by the gauge bosons $\mathcal{F}_{4,5}^{\mu}$ and $\mathcal{F}_{6,7}^{\mu}$ contribute to the taon leptonic decays $\tau \rightarrow e \nu_{\tau} \bar{\nu}_{e}$ and $\tau \rightarrow \mu \nu_{\tau} \bar{v}_{\mu}$ which rates are well consistent with the SM predictions [33]. Then, in the case $w_{1,2,3} \sim v_{\mathcal{F}}$ but $w_{1} \neq w_{2}$, the branching ratio $\Gamma\left(\tau \rightarrow \mu \nu_{\tau} \bar{v}_{\mu}\right) / \Gamma(\tau \rightarrow$ $\left.e \nu_{\tau} \bar{\nu}_{e}\right)$ can have order $G_{\mathcal{F}} / G_{F} \sim \delta_{\mu}$ deviation from the $\mathrm{SM}$ prediction which can be experimentally testable. For a comparison, the present experimental value of this ratio is $0.9762(28)$ [5], which is $1.3 \sigma$ larger than the SM predicted value 0.9726 . In addition, the terms in $\mathcal{L}_{\text {eff }}^{e v}$ with the diagonal generators $\lambda_{3}$ and $\lambda_{8}$ give rise to non-standard neutrino interactions with leptons. But respective coupling constants are of the order of $G_{\mathcal{F}}=\delta_{\mu} G_{F}$, and hence well below the experimental constraints.

The last term $\mathcal{L}_{\text {eff }}^{v v}$ in (31) contains the non-standard interactions between neutrinos, but present experimental limits on the neutrino self-interactions are very weak. However, second term $\mathcal{L}_{\text {eff }}^{e e}$ in (31) containing charged leptons in principle is testable for the scale $v_{\mathcal{F}}$ of few $\mathrm{TeV}$.

Interestingly, if the flavor eigenstates $e_{1}, e_{2}, e_{3}$ are the mass eigenstates $e, \mu, \tau$, the terms (31) do not contain any LFV operators inducing processes like $\mu \rightarrow 3 e$, $\tau \rightarrow 3 \mu$ etc. However, the lepton flavor-conserving contact operators $-\frac{4 \pi}{\Lambda_{L}^{2}}\left(\overline{e_{L}} \gamma_{\mu} e_{L}\right)^{2},-\frac{2 \pi}{\Lambda_{L}^{2}}\left(\overline{e_{L}} \gamma^{\mu} e_{L}\right)\left(\overline{\mu_{L}} \gamma_{\mu} \mu_{L}\right)$, etc. are restricted by the 'compositeness' limits $\Lambda_{L}^{-}($eeeee $)>$ $10.3 \mathrm{TeV}$ and $\Lambda_{L}^{-}(e e \mu \mu)>9.5 \mathrm{TeV}$. Comparing these operators with the corresponding terms in (31) and taking into account the relations (30), the 'compositeness' scales can be expressed in terms of the scale $v_{\mathcal{F}}$. Hence, we obtain the limit

$v_{\mathcal{F}}>\left(\frac{r+1}{r+0.5}\right)^{1 / 2} \times 2.1 \mathrm{TeV}$.

Here the $r$-dependent pre-factor approaches 1 when $r \gg 1$ and it becomes $\sqrt{2}$ in the opposite limit $r \ll 1$. Thus, the strongest limit emerges in the latter case, $v_{\mathcal{F}}>3 \mathrm{TeV}$ or so, which is anyway fulfilled for our benchmark range $v_{\mathcal{F}} \simeq$ $(6 \div 7) \mathrm{TeV}$.
The flavor eigenstates $e_{1}, e_{2}, e_{3}$ coincide with the mass eigenstates $e, \mu, \tau$, if the Yukawa matrix $Y_{e}^{i j}$ in (26) is diagonal. This can be achieved by imposing some additional discrete symmetries between the flavons $\eta_{i}$ and $\xi_{i}$ of $S U(3)_{\ell}$ and $S U(3)_{e}$ sectors which would forbid the non-diagonal terms $y_{i j}$ in operator (23). However, in general case the initial flavor basis of the LH leptons is related to the mass basis by the unitary transformation

$$
\left(\begin{array}{l}
e_{1} \\
e_{2} \\
e_{3}
\end{array}\right)_{L}=U_{L}\left(\begin{array}{l}
e \\
\mu \\
\tau
\end{array}\right)_{L}=\left(\begin{array}{ccc}
U_{1 e} & U_{1 \mu} & U_{1 \tau} \\
U_{2 e} & U_{2 \mu} & U_{2 \tau} \\
U_{3 e} & U_{3 \mu} & U_{3 \tau}
\end{array}\right)\left(\begin{array}{l}
e \\
\mu \\
\tau
\end{array}\right)_{L}
$$

Then, in the basis of mass eigenstates, the operators $\mathcal{L}_{\text {eff }}^{e e}$ read as in (31) but with the substitution $\lambda_{a} / x_{a} \rightarrow U^{\dagger}\left(\lambda_{a} / x_{a}\right) U$. Interestingly, in the limit $r=1$, i.e. when the VEVs $w_{1,2,3}$ are equal and so $x_{a}=1$, all flavor bosons $\mathcal{F}_{a}^{\mu}$ have equal masses, and the substitution $\lambda_{a} \rightarrow U^{\dagger} \lambda_{a} U$ is simply a basis redetermination of the Gell-Mann matrices. Therefore, no LFV effects will emerge in this case since the global $S O(8)_{\ell}$ symmetry acts as a custodial symmetry. Namely, by Fierz transformations, using also the Fierz identities for the GellMann matrices, we obtain

$$
-\frac{G_{\mathcal{F}}}{\sqrt{2}} \sum_{a=1}^{8}\left(\overline{\boldsymbol{e}_{L}} \gamma_{\mu} \lambda_{a} \boldsymbol{e}_{L}\right)^{2}=-\frac{4}{3} \frac{G_{\mathcal{F}}}{\sqrt{2}}\left(\overline{\boldsymbol{e}_{L}} \gamma_{\mu} \boldsymbol{e}_{L}\right)^{2}
$$

Obviously, the latter expression is invariant under the unitary transformation (33).

In general case $r \neq 1$, the mixing (33) gives rise to the LFV operators as e.g. the one inducing $\mu \rightarrow 3 e$ decay:

$$
\begin{aligned}
& -\frac{4 G_{\mu e e e}}{\sqrt{2}}\left(\overline{e_{L}} \gamma^{\mu} \mu_{L}\right)\left(\overline{e_{L}} \gamma^{\mu} e_{L}\right)+\text { h.c., } \\
& \frac{4 G_{\mu e e e}}{\sqrt{2}}=\frac{C(r)}{2 v_{\mathcal{F}}^{2}}\left[1+\frac{1-r}{r}\left|U_{3 e}\right|^{2}\right] U_{3 e}^{*} U_{3 \mu},
\end{aligned}
$$

where the function $C(r)=(r-1) r[(r+1)(r+0.5)]^{-1}$ is limited as $|C(r)|<1$, reaching the maximal value at $r \gg 1$, and it vanishes at $r=1$. Then, taking $\left|U_{3 e}\right| \ll 1$, we obtain for the branching ratio of $\mu \rightarrow 3 e$ decay

$$
\frac{\Gamma(\mu \rightarrow e e \bar{e})}{\Gamma\left(\mu \rightarrow e v_{\mu} \bar{v}_{e}\right)}=\frac{1}{2}\left|\frac{G_{\mu e e e}}{G_{F}}\right|^{2}=\frac{1}{8}\left(\delta_{\mu} C(r)\left|U_{3 e}^{*} U_{3 \mu}\right|\right)^{2}
$$

The experimental upper bound on this branching ratio is $10^{-12}$ [5]. Taking $\delta_{\mu}=\left(v_{\mathrm{w}} / v_{\mathcal{F}}\right)^{2}=7 \times 10^{-4}$, the limit $\delta_{\mu}\left|C U_{3 e}^{*} U_{3 \mu}\right| / \sqrt{8}<10^{-6}$ translates into $\left|C U_{3 e}^{*} U_{3 \mu}\right|<$ $0.4 \times 10^{-2}$ which is nicely satisfied if the lepton mixing angles in (33) are comparable with the CKM mixing angles in (1) or even larger. E.g. if the VEV ratio is in between $r=0.5 \div 1.5$, then $|C(r)|<1 / 7$ so that $\left|U_{3 e}^{*} U_{3 \mu}\right|<(1 / 6)^{2}$ or so would suffice for properly suppressing the $\mu \rightarrow 3 e$ decay rate. This means that in this case the matrix elements $\left|U_{3 \mu}\right|$ and $\left|U_{3 e}\right|$ can be almost as large as the Cabibbo angle 
$\sin \theta_{C}=V_{u s}$. The experimental limits on other LFV effects as e.g. $\tau \rightarrow 3 \mu$ are weaker, and following the lines of Ref. [31] one can show that in our model with $v_{\mathcal{F}} \simeq 6 \mathrm{TeV}$ or so, they are fulfilled even for whatever large mixings in (33). Once again, for $r=1$ all LFV effects are vanishing owing to custodial symmetry, see Eq. (34).

7. Let us discuss briefly how the hypothesis $G_{\mu} \neq G_{F}$ could affect the SM precision tests. In the SM, at tree level, the weak gauge boson masses are $M_{W}=g v_{\mathrm{w}} / \sqrt{2}=e v_{\mathrm{w}} / \sqrt{2} \sin \theta_{W}$ and $M_{Z}=M_{W} / \cos \theta_{W}$ where $\theta_{W}$ is the weak angle. For precision tests the radiative corrections are important which depend also on the top quark and Higgs mass.

The world averages of experimentally measured masses of $Z$ and $W$ reported by PDG 2018 are [5]:

$M_{Z}^{\exp }=91.1876(21) \mathrm{GeV}$,

$M_{W}^{\exp }=80.379(12) \mathrm{GeV}$,

while the SM global fit yields to the following values:

$M_{Z}^{\mathrm{SM}}=91.1884(20) \mathrm{GeV}$,

$M_{W}^{\mathrm{SM}}=80.358(4) \mathrm{GeV}$.

Hence, the theoretical and experimental values of $Z$-mass are in perfect agreement while for $W$-boson the two values have about $1.6 \sigma$ discrepancy:

$M_{W}^{\exp }-M_{W}^{\mathrm{SM}}=(21 \pm 13) \mathrm{MeV}$

In the SM the mass of $W$-boson, including radiative corrections, is determined as

$M_{W}=\frac{A_{0}}{\hat{s}_{Z}\left(1-\Delta \hat{r}_{W}\right)^{1 / 2}}$

where $A_{0}=\left(\pi \alpha / \sqrt{2} G_{F}\right)^{1 / 2}=37.28039(1) \mathrm{GeV}$ taking $G_{F}=G_{\mu}$, the factor $1-\Delta \hat{r}_{W}=0.93084(8)$ includes the main radiative corrections and $\hat{s}_{Z}^{2}=1.0348(2) s_{W}^{2}$ is the corrected value of $\sin ^{2} \theta_{W}\left(M_{Z}\right)$ by including the top and Higgs mass dependent corrections. The theoretical mass $M_{W}=80.358(4) \mathrm{GeV}$ (38) is then obtained by substituting in (40) the value $\hat{s}_{Z}^{2}=0.23122$ (3) obtained from the SM global fit [5]. In our scenario, however, $G_{F} \neq G_{\mu}$. Should we just set in $A_{0}$ instead of $G_{F}=G_{\mu}$ the "corrected" value $G_{F}=\left(1+\delta_{\mu}\right)^{-1} G_{\mu}$, then $A_{0}$ should be rescaled by a factor $\left(1+\delta_{\mu}\right)^{1 / 2}$, and correspondingly the "theoretical" value of $M_{W}$ (40) too. In particular, for $\delta_{\mu}=7 \times 10^{-4}$ we would get $M_{W}=80.386 \mathrm{GeV}$, right in the ball-park of the experimental values (38). However, this is not the right thing to do.

In the global fit of SM $M_{Z}$ is one of the input parameters with smallest experimental errors, along with the fine structure constant $\alpha$ and the "muon" Fermi constant $G_{\mu}$. Essentially, this is the main reason of the good coincidence between $M_{Z}^{\exp }$ and $M_{Z}^{\mathrm{SM}}$. In fact, the SM implies the relation

$M_{Z}=\frac{M_{W}}{\hat{c}_{Z} \hat{\rho}^{1 / 2}}=\frac{A_{0}}{\hat{s}_{Z} \hat{c}_{Z}\left(1-\Delta \hat{r}_{W}\right)^{1 / 2} \hat{\rho}^{1 / 2}}$ where $\hat{\rho}=1+\rho_{t}+\delta \rho=1.01013(5)$ includes the weak isospin breaking effects, dominantly from the quadratic $m_{t}$ dependent corrections $\rho_{t}=3 G_{F} m_{t}^{2} / 8 \sqrt{2} \pi^{2}$. Therefore, taking the experimental value of $Z$-mass (37), Eq. (41) can be used for determination of $\hat{s}_{Z}^{2}$ parameter, $\hat{s}_{Z}^{2}=$ 0.23123 (3). This, in turn, from $M_{W}=M_{Z} \hat{\rho}^{1 / 2} \hat{c}_{Z}$ gives $M_{W}=80.357(4)_{\mathrm{SM}} \mathrm{GeV}$, i.e. practically the same as the global fit result (38). This is because the determination of the parameter $\hat{s}_{Z}^{2}$ in the SM global fit is dominated by the results of $Z$-pole measurements.

However, in our scenario rescaling $A_{0} \rightarrow A_{0}(1+$ $\left.\delta_{\mu}\right)^{1 / 2}$ changes the value of $\hat{s}_{Z}^{2}$. In particular, taking $\delta_{\mu}=$ $(7.6 \pm 1.6) \times 10^{-4}$, we get $\hat{s}_{Z}^{2}=0.23148(3)_{\mathrm{SM}}(5)_{\delta_{\mu}}$. Then, again from $M_{W}=M_{Z} \hat{\rho}^{1 / 2} \hat{c}_{Z}$, we get $M_{W}=$ $80.344(4)_{S M}(3)_{\delta_{\mu}} \mathrm{GeV}$. Thus, unfortunately, while the effect is there, in reality it goes right to the opposite direction. So, our determination of $M_{W}$ differs from $M_{W}^{\mathrm{SM}}, M_{W}^{\mathrm{SM}}-M_{W}^{\text {our }}=$ $(13 \pm 3) \mathrm{MeV}$. Thus, with $M_{W}^{\mathrm{SM}}$ already being in tension with the experimental value (37), our result has more tension: $M_{W}^{\exp }-M_{W}^{\text {our }}=(35 \pm 13) \mathrm{MeV}(2.7 \sigma) .{ }^{4}$ If the tension will increase with future precision, this would mean that one has to admit at least some minimal step beyond the SM. The relation between $W$ and $Z$ masses can be improved by increasing of $\rho$-parameter via e.g. the $\mathrm{VEV} \sim 1 \mathrm{GeV}$ of a scalar triplet of the electroweak $S U(2) \times U(1)$, or by diminishing $Z$ mass by few $\mathrm{MeV}$ e.g. via its mixing with some extra gauge bosons like $Z^{\prime}$ at the $\mathrm{TeV}$ scale or perhaps also with the flavor gauge bosons considered in the previous section.

8. The value $\left|V_{u d}\right|$ can be extracted also from free neutron decay by combining the results on the measurements of the neutron lifetime $\tau_{n}$ with those of the axial current coupling constant $g_{A}$. The master formula reads (see e.g. in a recent review [34]):

$$
\begin{aligned}
\left|V_{u d}\right|^{2} & =\frac{K / \ln 2}{G_{F}^{2} \mathcal{F}_{n} \tau_{n}\left(1+3 g_{A}^{2}\right)\left(1+\Delta_{R}^{V}\right)} \\
& =\frac{5024.46(30) \mathrm{s}}{\tau_{n}\left(1+3 g_{A}^{2}\right)\left(1+\Delta_{R}^{V}\right)}
\end{aligned}
$$

where $\mathcal{F}_{n}=f_{n}\left(1+\delta_{R}^{\prime}\right)$ is the neutron $f$-value $f_{n}=$ 1.6887(1) corrected by the long-distance QED correction $\delta_{R}^{\prime}=0.01402(2)$ [35]. This equation, taking the values $\tau_{n}=880.2 \pm 1.0 \mathrm{~s}, g_{A}=1.2724 \pm 0.0023$ adopted in PDG 2018 [5], and $\Delta_{R}^{V}=0.02361$ (38) [7], would give the value

$\left|V_{u d}\right|=0.97577(55)_{\tau_{n}}(146)_{g_{A}}(18)_{\Delta_{R}^{V}}=0.97577(157)(43)$

which has an order of magnitude larger error than $\left|V_{u d}\right|=$ $0.97420(10)_{\mathcal{F}_{t}}(18)_{\Delta_{R}^{V}}=0.97420(21)$ obtained from (6) and used in (9), due to large uncertainties in $\tau_{n}$ and $g_{A}$.

4 Let us remark that the tension with the latest results of ATLAS $M_{W}^{\mathrm{ATL}}=80.370(19)$ is much weaker $(1.3 \sigma), M_{W}^{\mathrm{ATL}}-M_{W}^{\text {our }}=$ $(26 \pm 20) \mathrm{MeV}$. 


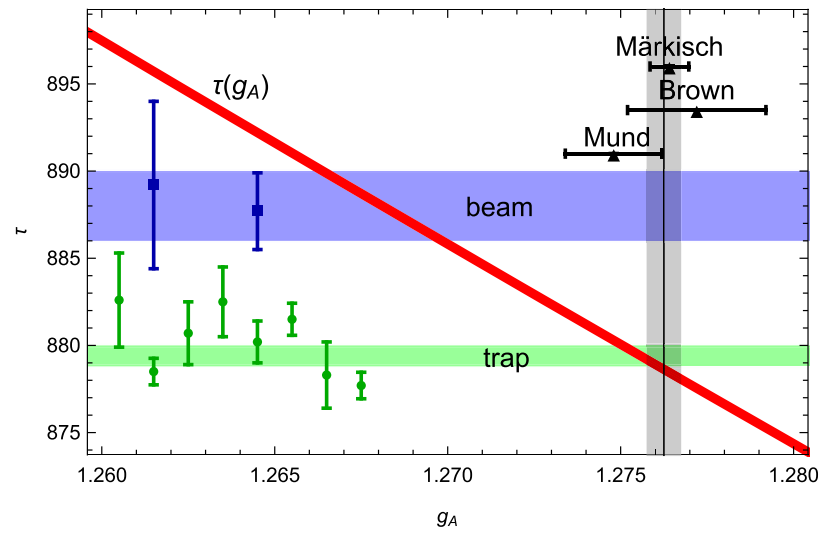

Fig. 7 The red band shows the precision relation (44) between $g_{A}$ and $\tau_{n}$. Black triangles with horizontal error bars show values of $g_{A}$ reported in Refs. [37-39] and vertical grey band corresponds to their average (45). Green circles show values of $\tau_{n}$ reported by trap experiments [4250] with respective error bars and horizontal green band shows their average (47). Blue squares and blue horizontal band show the the same for beam experiments [51,52]

However, rather than for determination of $\left|V_{u d}\right|$, Eq. (42) can be used for a consistency check. Namely, by comparing it with Eq. (6) we get a relation between $\tau_{n}$ and $g_{A}$ [36]:

$\tau_{n}=\frac{2 \mathcal{F} t}{\ln 2 \mathcal{F}_{n}\left(1+3 g_{A}^{2}\right)}=\frac{5172.0(1.1) \mathrm{s}}{1+3 g_{A}^{2}}$

In Fig. 7 this relation is shown by the red band. This formula is very accurate since the common factors in Eqs. (6) and (42) including the Fermi constant and radiative corrections $\Delta_{R}^{V}$ cancel out.

For the axial current coupling $g_{A}$, the PDG 2018 quotes the value $g_{A}=1.2724 \pm 0.0023$. However, the results of the latest and most accurate experiments [37-39] which measured $\beta$-asymmetry parameter using different techniques (the cold neutrons in PERKEO II and PERKEO III experiments $[37,39]$ and ultra-cold neutrons in the UCNA experiment [38]), are in perfect agreement among each other, and their average determines the axial current coupling $g_{A}$ with impressive (better than one per mille) precision:

$g_{A}=1.27625 \pm 0.00050$.

Figure 7 shows the results of Refs. [37-39] and their average (vertical grey band). For $g_{A}$ in this range Eq. (44) gives the Standard Model prediction for the neutron lifetime

$\tau_{n}^{\mathrm{SM}}=878.7 \pm 0.6 \mathrm{~s}$

From the experimental side, the neutron lifetime is measured in two types of experiments. The trap experiments measure the disappearance rate of the ultra-cold neutrons (UCN) by counting the survived neutrons after storing them for different times in the UCN traps and determine the neutron decay width $\Gamma_{n}=\tau_{n}^{-1}$. The beam experiments are the appearance experiments, measuring the width of $\beta$-decay $n \rightarrow p e \bar{v}_{e}, \Gamma_{\beta}=\tau_{\beta}^{-1}$, by counting the produced protons in the monitored beam of cold neutrons. In the Standard Model the neutron decay should always produce a proton, and so both methods should measure the same value $\Gamma_{n}=\Gamma_{\beta}$.

However, there is tension between the results obtained using these two methods, which was pointed out in Refs. [40,41]. Figure 7 clearly demonstrates the discrepancy. Namely, by averaging the presently available results of eight trap experiments [42-50] one obtains: ${ }^{5}$

$\tau_{\text {trap }}=879.4 \pm 0.6 \mathrm{~s}$,

which is compatible with $\tau_{n}^{\mathrm{SM}}$ (46). In particular, this value of $\tau_{n}$ together with new $g_{A}$ (45) and new value $\Delta_{R}^{V}=$ 0.02467(22) [8], determines $\left|V_{u d}\right|$ with the precision more than 3 times better than in (43):

$\left|V_{u d}\right|=0.97327(33)_{\tau_{n}}(32)_{g_{A}}(10)_{\Delta_{R}^{V}}=0.97327(47)$.

This is compatible with $\left|V_{u d}\right|=0.97370(10)_{\mathcal{F}_{t}}(10)_{\Delta_{R}^{V}}=$ 0.97370 (14) from supeallowed $0^{+}-0^{+}$decays used in (10) but has 3 times larger error than the latter. For making it competitive with the latter determination, the neutron lifetime should be measured with precision of $0.1 \mathrm{~s}$ and $g_{A}$ with precision 3 times better than in (45), which can be realistic in future experiments.

On the other hand, the beam experiments [51,52] yield the value

$\tau_{\text {beam }}=888.0 \pm 2.0 \mathrm{~s}$

which is $4.4 \sigma$ away from the SM prediction (46). Therefore, it is more likely that the true value of the neutron lifetime is the one measured by trap experiments (47) which is consistent with the SM prediction (46).

About 1 per cent deficit of produced protons in the beam experiments [51,52] might be due to some unfixed systematic errors. Alternatively, barring the possibility of uncontrolled systematics and considering the problem as real, a new physics must be invoked which could explain about one per cent deficit of protons produced in the beam experiments. One interesting possibility can be related to the neutronmirror neutron $\left(n-n^{\prime}\right)$ oscillation [53-55], provided that ordinary and mirror neutrons have a tiny mass difference 300 neV or so [56]. Then in large magnetic fields (5 Tesla) used in beam experiments $n-n^{\prime}$ conversion probability can be resonantly enhanced to about $\sim 0.01$, and the corresponding fraction of neutrons converted in mirror neutrons will decay

\footnotetext{
5 The PDG 2018 average $\tau_{n}=880.2 \pm 1.0 \mathrm{~s}$ includes the results of five trap experiments [42-47] and two beam experiments [51,52]. The error enlarged by a factor $\sqrt{\chi_{\text {dof }}^{2}} \approx 2$, essentially for a loose compatibility between the data obtained from the trap and the beam experiments. This average does not include the results of three recent trap experiments [48-50] published in 2018.
} 
in an invisible (mirror) channel without producing ordinary protons.

Concluding this section, let us remark that the recent accurate calculations of the short-range radiative corrections $\Delta_{R}^{V}$ $[8,12]$ and respective redetermination of $V_{u d}$ has no influence on the determination of $\tau_{n}$ (46) obtained from Eq. (44). In fact, the latter equation directly relates the neutron lifetime to the value $\mathcal{F} t$ accurately measured in superallowed $0^{+}-0^{+}$nuclear transitions and to the value $g_{A}$ obtained from accurate measurements of $\beta$-asymmetry. Notice that the relation (44) remains valid also in the presence of nonstandard vector $G_{V}$ or axial $G_{A}$ coupling constants (which can be the case if some non-standard interactions mediated by new vector bosons also contribute to the neutron decay) since the value $G_{V}$ (independently whether it is equal to $G_{F}\left|V_{u d}\right|$ or not) anyway cancels out [57]. Hence, only the ratio $g_{A}=G_{A} / G_{V}$ remains relevant which is accurately determined from the measurements of $\beta$-asymmetry. In particular, Eq. (44) remains valid in our model with $G_{F} \neq G_{\mu}$ discussed in previous section.

9. In this paper we discussed the CKM unitarity problem. The present experimental and theoretical accuracy in the determination of the first row elements in the CKM matrix (1) indicates towards $4.3 \sigma$ deviation from the unitarity (2). We investigated two new physics scenarios which could settle the problem. The respective results are summarized in Table 1.

The first, rather straightforward possibility is related to the existence of extra weak isosinglet down-type quark $b^{\prime}$ with the mass of few $\mathrm{TeV}$ which should have a rather large mixing with the first family, $\left|V_{u b^{\prime}}\right| \simeq 0.04$. However, apart of the persistent question "who has ordered that?", this scenario has some unnatural features related to the flavor-changing phenomena. In particular, given that $\left|V_{u b^{\prime}}\right| \simeq 0.04$, then $b^{\prime}$ will induce too large effects in $K^{0}-\bar{K}^{0}$ system etc. unless its mixings with 2nd and 3rd families $V_{c b^{\prime}}$ and $V_{t b^{\prime}}$ are rather small. Perhaps such a situation is possible by some conspiracies, but a priori it looks rather weird.

As another possibility for restoring unitarity, one can introduce additional effective operator contributing to the muon decay in positive interference with the Standard Model contribution. In this case the Fermi constant would be slightly different from muon decay constant, $G_{F}=G_{\mu} /\left(1+\delta_{\mu}\right)$, where $\delta_{\mu} \simeq 7 \times 10^{-4}$ would suffice for unitarizing the CKM matrix. Namely, the values of $V_{u s}$ and $V_{u d}$ (which are normally extracted by assuming $G_{F}=G_{\mu}$ ) are shifted by a factor $1+\delta_{\mu}$ while their ratio is not affected. The needed effective operator can be mediated by a flavor changing boson of a gauge horizontal symmetry $S U(3)_{\ell}$ between the three lepton families which is spontaneously broken at the scale of few TeV.
The scenario with gauge inter-family symmetry $S U(3)_{\ell} \times$ $S U(3)_{e}$ acting on left-handed and right-handed leptons can give a natural understanding of the mass hierarchy among charged leptons and large mixing of neutrinos as a consequence of spontaneous breaking pattern of this symmetry. Interestingly, despite the fact that these gauge bosons have flavor-changing couplings with the leptons, their exchanges do not induce dramatic LFV effects as e.g. $\mu \rightarrow 3 e, \tau \rightarrow 3 \mu$ decays etc. since these effects can be kept under control thanks to approximate custodial symmetry.

Analogously, one can consider the inter-family gauge symmetry $S U(3)_{Q} \times S U(3)_{u} \times S U(3)_{d}$ between the quarks. Its breaking pattern can be at the origin of the quark mass and mixing spectrum. Interestingly, the flavor-changing gauge bosons of $S U(3)_{Q}$ can contribute to the hadronic decays of kaons, hyperons, etc. In supersymmetric extension of the SM, the chiral gauge symmetries $S U(3)_{\ell} \times S U(3)_{e}$ for leptons and $S U(3)_{Q} \times S U(3)_{u} \times S U(3)_{d}$ for quarks can be also motivated as a natural tool for realizing the minimal flavor violation scenario [58-60].

There is the interesting possibility that these flavor gauge symmetries are common symmetries between particles of ordinary and mirror sectors, which is also motivated by the possibility of cancellation of triangle anomalies of gauge $S U$ (3) factors between the ordinary and mirror fermions [61]. Mirror matter is also a viable candidate for dark matter (see e.g. reviews [62-64]). Since flavor gauge bosons are messengers between the two sectors, they can mediate new flavor violating phenomena such as muonium-mirror muonium, kaon-mirror kaon oscillations, etc. [31] and also can give a possible portal for direct detection of mirror matter in dark matter detectors $[65,66]$. Cosmological implications and limits were discussed in Ref. [31].

Acknowledgements The work of ZB was supported by the research Grant "The Dark Universe: A Synergic Multimessenger Approach" No. 2017X7X85K under the program PRIN 2017 funded by the Ministero dell'Istruzione, Università e della Ricerca (MIUR). The work of RB and ZB was supported in part by Shota Rustaveli National Science Foundation (SRNSF) of Georgia, Grant DI-18-335/New Theoretical Models for Dark Matter Exploration. ZB and BB thank the Galileo Galilei Institute (GGI) for Theoretical Physics for its hospitality and support.during the Workshop "Next Frontiers in the Search for Dark Matter" where the final part of this work was done.

Data Availability Statement This manuscript has no associated data or the data will not be deposited. [Authors' comment: This is a theoretical work. No experimental data were used.]

Open Access This article is licensed under a Creative Commons Attribution 4.0 International License, which permits use, sharing, adaptation, distribution and reproduction in any medium or format, as long as you give appropriate credit to the original author(s) and the source, provide a link to the Creative Commons licence, and indicate if changes were made. The images or other third party material in this article are included in the article's Creative Commons licence, unless indicated otherwise in a credit line to the material. If material is not 
included in the article's Creative Commons licence and your intended use is not permitted by statutory regulation or exceeds the permitted use, you will need to obtain permission directly from the copyright holder. To view a copy of this licence, visit http://creativecomm ons.org/licenses/by/4.0/.

Funded by SCOAP ${ }^{3}$.

\section{References}

1. J.C. Hardy, I.S. Towner, Phys. Rev. C 91(2), 025501 (2015). arXiv:1411.5987 [nucl-ex]

2. J. Hardy, I.S. Towner, PoS CKM 2016, 028 (2016)

3. V. Tishchenko et al., [MuLan Collaboration], Phys. Rev. D 87(5), 052003 (2013). arXiv:1211.0960 [hep-ex]

4. M. Moulson, PoS CKM 2016, 033 (2017). arXiv: 1704.04104 [hepex]

5. M. Tanabashi et al., [Particle Data Group], Phys. Rev. D 98(3), $030001(2018)$

6. S. Aoki et al., [FLAG], Eur. Phys. J. C 77(2), 112 (2017). arXiv:1607.00299 [hep-lat]

7. W.J. Marciano, A. Sirlin, Phys. Rev. Lett. 96, 032002 (2006). arXiv:hep-ph/0510099

8. C.Y. Seng, M. Gorchtein, H.H. Patel, M.J. Ramsey-Musolf, Phys. Rev. Lett. 121(24), 241804 (2018). arXiv:1807.10197 [hep-ph]

9. A. Bazavov et al., [Fermilab Lattice and MILC Collaborations], Phys. Rev. D 99(11), 114509 (2019). arXiv:1809.02827 [hep-lat]

10. S. Aoki et al., [Flavour Lattice Averaging Group]. arXiv:1902.08191 [hep-lat]

11. M. Di Carlo, D. Giusti, V. Lubicz et al., Phys. Rev. D 100(3), 034514 (2019). arXiv:1904.08731 [hep-lat]

12. A. Czarnecki, W.J. Marciano, A. Sirlin, Phys. Rev. D 100(7), 073008 (2019). arXiv:1907.06737 [hep-ph]

13. E. Kuflik, Y. Nir, T. Volansky, Phys. Rev. Lett. 110(9), 091801 (2013). arXiv:1204.1975 [hep-ph]

14. B. Belfatto, Z. Berezhiani (in preparation)

15. L. Lavoura, J.P. Silva, Phys. Rev. D 47, 1117 (1993)

16. Z.G. Berezhiani, Phys. Lett. B 129, 99 (1983)

17. Z.G. Berezhiani, Phys. Lett. B 150, 177 (1985)

18. Z.G. Berezhiani, J.L. Chkareuli, JETP Lett. 35, 612 (1982)

19. Z.G. Berezhiani, J.L. Chkareuli, Sov. J. Nucl. Phys. 37, 618 (1983)

20. Z.G. Berezhiani, J.L. Chkareuli, Sov. J. Nucl. Phys. 52, 383 (1990)

21. Z.G. Berezhiani, M.Y. Khlopov, Z. Phys. C 49, 73 (1991)

22. Z.G. Berezhiani, M.Y. Khlopov, Sov. J. Nucl. Phys. 51, 739 (1990)

23. Z.G. Berezhiani, M.Y. Khlopov, Sov. J. Nucl. Phys. 52, 60 (1990)

24. Z. Berezhiani, A. Rossi, Nucl. Phys. B 594, 113 (2001). arXiv:hep-ph/0003084

25. S.F. King, G.G. Ross, Phys. Lett. B 574, 239 (2003). arXiv:hep-ph/0307190

26. Z. Berezhiani, F. Nesti, JHEP 0603, 041 (2006). arXiv:hep-ph/0510011

27. P. Binetruy, S. Lavignac, P. Ramond, Nucl. Phys. B 477, 353 (1996). arXiv:hep-ph/9601243

28. E. Dudas, C. Grojean, S. Pokorski, C.A. Savoy, Nucl. Phys. B 481, 85 (1996). arXiv:hep-ph/9606383

29. Z. Berezhiani, Z. Tavartkiladze, Phys. Lett. B 396, 150 (1997). arXiv:hep-ph/9611277

30. Z. Berezhiani, Z. Tavartkiladze, Phys. Lett. B 409, 220 (1997). arXiv:hep-ph/9612232

31. B. Belfatto, Z. Berezhiani, Eur. Phys. J. C 79(3), 202 (2019). arXiv:1812.05414 [hep-ph] (see also B. Belfatto, PoS EPS HEP2017, 660 (2017))
32. Z. Berezhiani, G.R. Dvali, Phys. Lett. B 450, 24 (1999). arXiv:hep-ph/9811378

33. A. Pich, Prog. Part. Nucl. Phys. 75, 41 (2014). arXiv:1310.7922 [hep-ph]

34. M. Gonzalez-Alonso, O. Naviliat-Cuncic, N. Severijns, Prog. Part. Nucl. Phys. 104, 165 (2019). arXiv:1803.08732 [hep-ph]

35. I.S. Towner, J.C. Hardy, Rep. Prog. Phys. 73, 046301 (2010)

36. A. Czarnecki, W.J. Marciano, A. Sirlin, Phys. Rev. Lett. 120, 202002 (2018). arXiv:1802.01804 [hep-ph]

37. D. Mund et al., Phys. Rev. Lett. 110, 172502 (2013). arXiv:1204.0013 [hep-ex]

38. M.A.-P. Brown et al., Phys. Rev. C 97(3), 035505 (2018). arXiv:1712.00884 [nucl-ex]

39. B. Märkisch et al., Phys. Rev. Lett. 122(24), 242501 (2019). arXiv:1812.04666 [nucl-ex]

40. A. Serebrov, A. Fomin, Phys. Procedia 17, 19 (2011)

41. G.L. Greene, P. Geltenbort, Sci. Am. 314, 36 (2016)

42. W. Mampe et al., JETP Lett. 57, 82 (1993)

43. A.P. Serebrov et al., Phys. Lett. B 605, 72 (2005). arXiv:nucl-ex/0408009

44. A.P. Serebrov et al., Phys. Rev. C 78, 035505 (2008). arXiv:nucl-ex/0702009 [NUCL-EX]

45. A. Pichlmaier et al., Phys. Lett. B 693, 221 (2010)

46. A. Steyerl et al., Phys. Rev. C 85, 065503 (2012)

47. S. Arzumanov et al., Phys. Lett. B 745, 79 (2015)

48. A.P. Serebrov et al., Phys. Rev. C 97(5), 055503 (2018). arXiv:1712.05663 [nucl-ex]

49. V.F. Ezhov et al., JETP 107, 11 (2018). arXiv:1412.7434 [nucl-ex]

50. R.W. Pattie Jr. et al., Science 360(6389), 627 (2018). arXiv:1707.01817 [nucl-ex]

51. J. Byrne et al., Europhys. Lett. 33, 187 (1996)

52. A.T. Yue et al., Phys. Rev. Lett. 111(22), 222501 (2013). arXiv:1309.2623 [nucl-ex]

53. Z. Berezhiani, L. Bento, Phys. Rev. Lett. 96, 081801 (2006). arXiv:hep-ph/0507031

54. Z. Berezhiani, L. Bento, Phys. Lett. B 635, 253 (2006) arXiv:hep-ph/0602227

55. Z. Berezhiani, Eur. Phys. J. C 64, 421 (2009). arXiv:0804.2088 [hep-ph]

56. Z. Berezhiani, Eur. Phys. J. C 79(6), 484 (2019). arXiv: 1807.07906 [hep-ph]

57. Z. Berezhiani, LHEP 158, 1 (2019). https://doi.org/10.31526/ LHEP.1.2019.118. arXiv:1812.11089 [hep-ph]

58. A. Anselm, Z. Berezhiani, Nucl. Phys. B 484, 97 (1997). arXiv:hep-ph/9605400

59. Z. Berezhiani, A. Rossi, Nucl. Phys. Proc. Suppl. 101, 410 (2001). arXiv:hep-ph/0107054

60. G. D'Ambrosio, G.F. Giudice, G. Isidori, A. Strumia, Nucl. Phys. B 645, 155 (2002). arXiv:hep-ph/0207036

61. Z. Berezhiani, Phys. Lett. B 417, 287 (1998)

62. Z. Berezhiani, Int. J. Mod. Phys. A 19, 3775 (2004)

63. Z. Berezhiani, Through the looking-glass: Alice's adventures in mirror world. In eds. by M. Shifman et al. I. Kogan Memorial Volume From Fields to Strings, Circumnavigating Theoretical Physics, vol 3 (World Scientific, 2005), pp. 2147-2195. arXiv:hep-ph/0508233

64. Z. Berezhiani, Eur. Phys. J. ST 163, 271 (2008)

65. R. Cerulli et al., Eur. Phys. J. C 77(2), 83 (2017). arXiv: 1701.08590 [hep-ex]

66. A. Addazi et al., Eur. Phys. J. C 75(8), 400 (2015). arXiv:1507.04317 [hep-ex] 